\title{
Imaging conundrum - COVID-related lung changes or connective tissue disease-related interstitial lung disease
}

\author{
Surabhi Vyas ${ }^{A, B, C, D, E, F}$, Ashu S. Bhalla $a^{A, B, C, E}$ \\ Department of Radiodiagnosis, All India Institute of Medical Sciences, New Delhi, India
}

\begin{abstract}
Dear Editor,
Coronavirus disease 2019 (COVID-19) pneumonia presents with clinical features of variable severity. Imaging in the form of chest radiograph and high-resolution computed tomography (HRCT) play a central role in the management of patients suffering from COVID-19 pneumonia. The HRCT findings in such cases reflect the lung injury caused due to the viral pneumonia and the results of a cascade of inflammatory mediators. In the initial phase of the disease (day 1-6 of illness), changes are described as subpleural ground glass opacities and consolidation, either alone or in combination with interlobular septal lines, which may give rise to the "crazy paving appearance". It is in the later phase of the disease (day 6-11 of illness) that the imaging appearance evolves into architectural distortion, perilobular opacities, and subpleural linear/curvilinear changes [1]. These appearances are similar to the imaging appearance of interstitial lung disease (ILD) patterns of organizing pneumonia (OP) and non-specific interstitial pneumonia (NSIP), which is an imaging conundrum. These imaging patterns are common HRCT findings in various connective tissue diseases (CTD)-related ILD. NSIP is the commonest pattern of CTD-related ILD, with systemic sclerosis being the archetype [2]. Rheumatoid arthritis (RA)-related ILD also shows an NSIP pattern, even though the pattern most frequently reported is the usual interstitial pneumonia (UIP). The OP pattern is commonly seen in dermatomyositis/polymyositis (PM/DM), and less commonly in RA, Sjögren syndrome, and systemic lupus erythematosus [3]. Because the presence of pre-existing ILD is a WHOdefined risk factor for severe affection of COVID-19,
\end{abstract}

these patients often present with HRCT appearances of their pre-existing disease and overlapping features of COVID pneumonia. The differentiation between the two becomes especially challenging in a resource constrained setting, where prior imaging for comparison may not be readily available.

The HRCT appearance of NSIP is that of lower lobe, predominantly peripheral changes, which are ground glass opacities (GGO) without bronchiectasis in cellular NSIP; GGO with fine reticular changes, microcysts, areas of interlobular septal thickening, tractional bronchiectasis, and volume loss in the fibrotic NSIP. The subpleural sparing described as a characteristic finding of NSIP, if absent, does not preclude the diagnosis. A lower lobar volume loss often accompanies the fibrotic NSIP [4]. Similar changes are also seen in patients of COVID-19 pneumonia [5] (Figure 1A, C).

Organizing pneumonia, on the other hand, is seen as patchy opacities in the peripheral basal lungs with characteristic perilobular distribution on HRCT [6] (Figure $1 B, D)$. Similar imaging features have been reported in a patient who received remdesivir and showed clinical recovery from COVID-19 pneumonia, with partial resolution of the HRCT features on follow-up [7].

We wish to highlight the similarity of CT features seen in these CTD-related ILDs and COVID-19-related pulmonary changes, especially in late and recovering stages. We also wish to emphasise the importance of complete history regarding a co-existing pulmonary condition due to CTD and evaluation of prior and follow-up imaging to understand the temporal relationship of the pulmonary changes and their resolution.

Correspondence address:

Dr Surabhi Vyas, Additional Professor, Department of Radiodiagnosis, All India Institute of Medical Sciences, Ansari Nagar, New Delhi 110029, India,

e-mail: surabhi_vyas@yahoo.com

Authors' contribution:

A Study design · B Data collection · C Statistical analysis · D Data interpretation · E Manuscript preparation · F Literature search · G Funds collection 

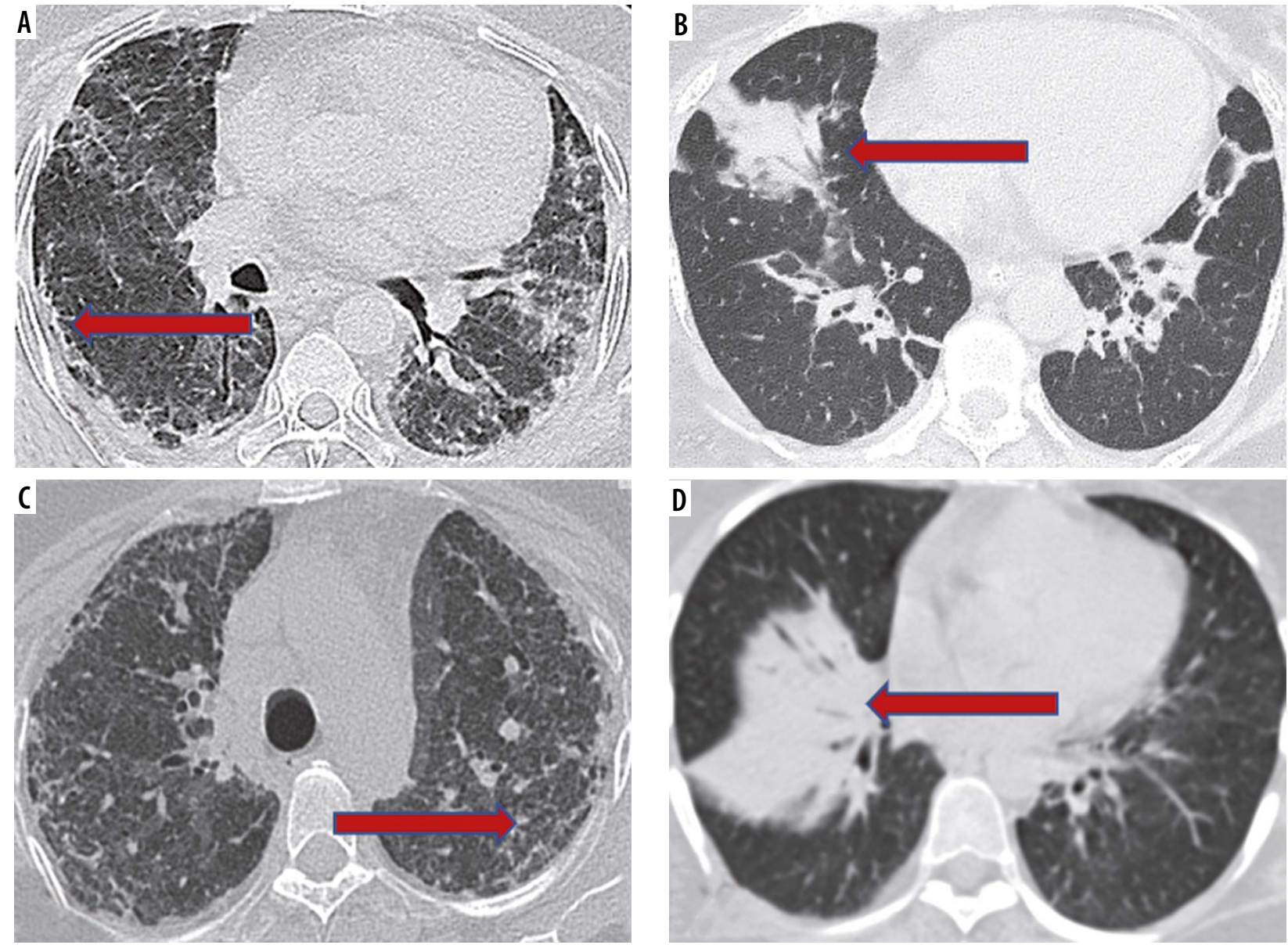

Figure 1. Imaging similarities between COVID-related pulmonary changes (A, B) and connective tissue disease (CTD)-related interstitial lung disease (ILD) (C, D). High-resolution computed tomography (HRCT) images of different patients of COVID-19 pneumonia showing subpleural curvilinear fibrotic changes and reticulations (arrow, A), and peribronchial consolidation (arrow, B). HRCT findings of subpleural reticulations, microcysts, and bronchiolectasis in a case of systemic sclerosis (arrow, C); organizing pneumonia in a case of polymyositis (arrow, D)

\section{Conflict of interest}

The authors report no conflict of interest.

\section{References}

1. Wang Y, Dong C, Hu Y, et al. Temporal changes of CT findings in 90 patients with COVID-19 pneumonia: a longitudinal study. Radiology 2020; 296: E55-E64.

2. Desai SR, Veeraraghavan S, Hansell DM, et al. CT features of lung disease in patients with systemic sclerosis: comparison with idiopathic pulmonary fibrosis and nonspecific interstitial pneumonia. Radiology 2004; 232: 560-567.

3. Silva CI, Muller NL. Interstitial lung disease in the setting of collagen vascular disease. Semin Roentgenol 2010; 45: 22-28.

4. Kligerman SJ, Groshong S, Brown KK, Lynch DA. Nonspecific interstitial pneumonia: radiologic, clinical, and pathologic considerations. Radiographics 2009; 29: 73-87.
5. Orlandi M, Lepri G, Bruni C, et al. The systemic sclerosis patient in the COVID-19 era: the challenging crossroad between immunosuppression, differential diagnosis and long-term psychological distress. Clin Rheumatol 2020; 39: 2043-2047.

6. Kligerman SJ, Franks TJ, Galvin JR. From the radiologic pathology archives: organization and fibrosis as a response to lung injury in diffuse alveolar damage, organizing pneumonia, and acute fibrinous and organizing pneumonia. Radiographics 2013; 33: 1951-1975

7. Pogatchnik BP, Swenson KE, Sharifi H, Bedi H, Berry GJ, Guo HH. Radiology-pathology correlation in recovered COVID-19, demonstrating organizing pneumonia. Am J Respir Crit Care Med 2020; 202: 598-599. 